\title{
Pulse-mode measurement of electron beam halo using diamond-based detector
}

\author{
Hideki Aoyagi, ${ }^{1}$ Yoshihiro Asano, ${ }^{2}$ Toshiro Itoga,${ }^{1}$ Nobuteru Nariyama, ${ }^{1}$ Teruhiko Bizen, ${ }^{1}$ \\ Takashi Tanaka, ${ }^{2}$ and Hideo Kitamura ${ }^{2}$ \\ ${ }^{1}$ Japan Synchrotron Radiation Research Institute (JASRI), 1-1-1 Kouto, Sayo-cho, Sayo-gun, Hyogo 679-5198, Japan \\ ${ }^{2}$ RIKEN SPring-8 Center, 1-1-1 Kouto, Sayo-cho, Sayo-gun, Hyogo 679-5148, Japan
}

(Received 16 September 2011; published 6 February 2012)

\begin{abstract}
Using a diamond-based detector, the electron beam halo in a high-energy accelerator can be measured with a lower detection limit than that using other instruments, such as a core monitor, a dose meter, or an optical fiber. We have successfully measured an electron beam halo using diamond-based detectors operating in the ionization mode, which were installed in the beam duct to measure the intensity of the beam halo directly. Pulse-by-pulse measurements were adopted to suppress the background noise efficiently. Feasibility tests on the diamond-based detector and beam halo monitor were performed in the beam dump area of the $8 \mathrm{GeV}$ SPring- 8 synchrotron booster and at the $250 \mathrm{MeV}$ SPring- 8 Compact SASE Source test accelerator for the SPring-8 Angstrom Compact free electron LAser (SACLA), respectively. We achieved a lower detection limit of $2 \times 10^{3}$ electrons/pulse for single-shot measurement, which corresponds to a ratio of about $10^{-6}$ relative to the typical charge of the beam core of $0.3 \mathrm{pC}$. We also confirmed the feasibility of the electron beam halo monitor for use as an interlock sensor to protect undulator permanent magnets used in SACLA from radiation damage.
\end{abstract}

DOI: 10.1103/PhysRevSTAB.15.022801

PACS numbers: 41.85.Qg, 29.40.Wk, 81.05.ug, 41.60.Cr

\section{INTRODUCTION}

Measurement of the electron beam halo in particle accelerators is a very important issue because it may degrade the quality of the electron beam and cause radio activation of the beam ducts and components. Furthermore, the beam halo may cause the demagnetization of undulator magnets, particularly those in x-ray free electron laser and synchrotron radiation facilities [1]. A diamond-based detector is one of the most promising candidates for measuring the intensity of high-energy electrons. Advantages of the diamond are high radiation hardness, high heat resistance, high insulation resistance, and so on [2]. Demonstration of the observation of charged particles using a diamond-based detector has been carried out at some facilities [3], and such detectors have been used operationally at the Large Hadron Collider (LHC) at CERN for a wide variety of beam instrumentation, such as particle counters, phase monitors, beam-loss monitors, and spectrometers [4,5]. Beam halo monitors using diamond-based detectors for observing charged particle beams directly inside the vacuum chamber, however, have not yet been introduced at any facilities until now.

We have adopted diamond-based detectors, which are made of chemical vapor-deposition diamond [6], for the detector head of a beam halo monitor. Diamond-based detectors are operated in the ionization mode (Fig. 1).

Published by the American Physical Society under the terms of the Creative Commons Attribution 3.0 License. Further distribution of this work must maintain attribution to the author(s) and the published article's title, journal citation, and DOI.
Electron-hole pairs are generated by the passage of a minimum ionization particle (MIP) through the diamond bulk. The structure of the diamond-based detector, which was fabricated by Kobe Steel, Ltd., is shown in Fig. 2. The dimensions of the diamond plate are $10 \mathrm{~mm}$ by $26 \mathrm{~mm}$, and the thickness is $0.3 \mathrm{~mm}$. A pair of aluminum electrodes on both sides of the plate is formed by a lithography technique. One electrode is used for signal reading and the other is used for applying a bias voltage. The active area of the diamond-based detector is the bottom part of the plate between the electrodes. The electron-hole pairs that are created in the active area can be induced toward the electrodes. The cross section of this active area has a size of $5 \mathrm{~mm}$ by $1 \mathrm{~mm}$. The thickness of the diamond is $0.3 \mathrm{~mm}$. If an electron having an energy of $8 \mathrm{GeV}$ is induced in the diamond, about $0.1 \%$ of its energy is absorbed in the diamond bulk on average. This detector has a self-sustaining structure and is not mounted on a package. Therefore, the active area can be placed closer to the beam center. The dark current is typically on the order of $100 \mathrm{pA}$ at a bias voltage of $100 \mathrm{~V}$. In the case of pulse-mode measurements, the dark

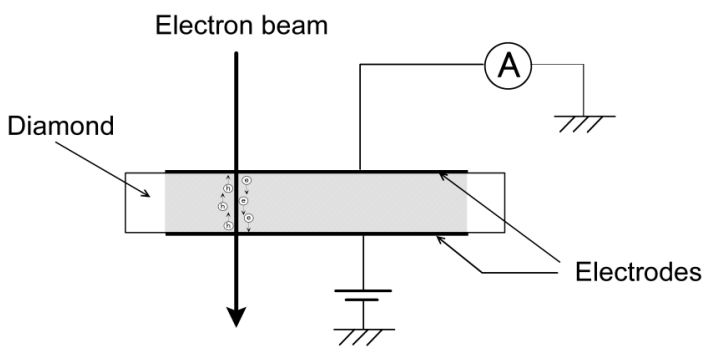

FIG. 1. Principle of the diamond-based detector. 


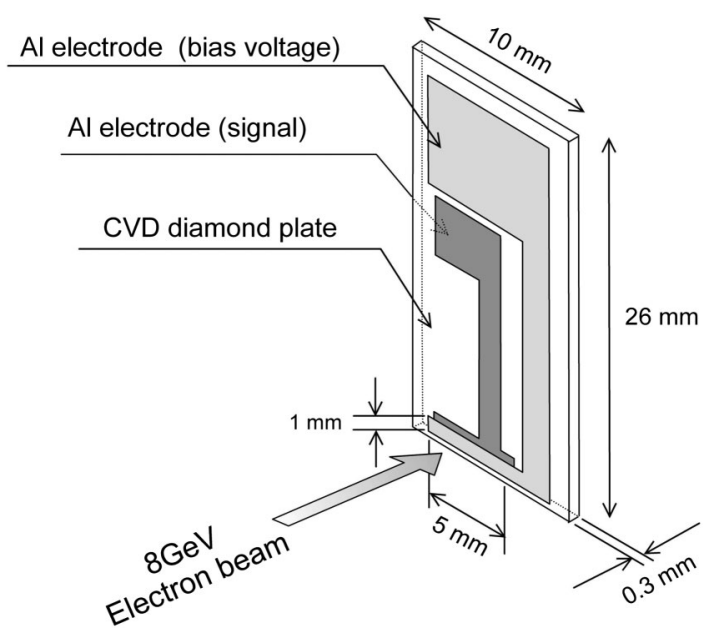

FIG. 2. Structure of the diamond-based detector.

current does not affect the output signal, because the charge from the dark current per pulse is negligibly small.

\section{EVALUATION OF THE DIAMOND-BASED DETECTORS}

To evaluate the basic characteristics of the diamondbased detectors, such as detection sensitivity to an electron beam and linearity, beam tests were performed at the beam dump of the $8 \mathrm{GeV}$ SPring- 8 booster synchrotron $[7,8]$. A schematic diagram of the setup used is shown in Fig. 3. An oscilloscope with a sampling rate of $20 \mathrm{GS} / \mathrm{s}$ and an analogue bandwidth of $4 \mathrm{GHz}$ was used. The coaxial cable has a certain dispersion, which gets less effective for short cables. We prepared low-attenuation cables with the length of $20 \mathrm{~m}$ because the oscilloscope must be set outside of the machine tunnel and the cable must be longer than about $20 \mathrm{~m}$. Coaxial cables with an impedance of $50 \Omega$ were used for impedance matching. The intrinsic pulse width of the detector signal is restricted by the RC time constant. The distributed capacitance must be minimized. We used as little wire as possible between the electrodes on the

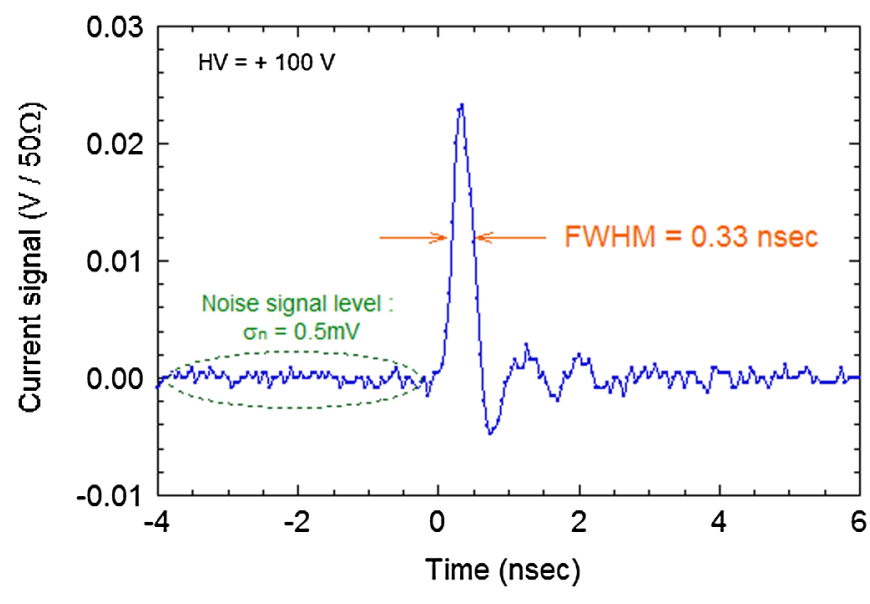

FIG. 4. Pulse shape of current signal of the diamond-based detector.

diamond-based detector and the subminiature type A connectors of the coaxial cables. This treatment also prevents the deformation of the pulse shape.

The typical pulse shape of the output signal is shown in Fig. 4. One-shot measurements were performed in the tests. The bias voltage was $+100 \mathrm{~V}$. The number of electrons per pulse was about $10^{4}$. A pulse length with a full width at half maximum (FWHM) of 0.33 ns was obtained. We used as little cable as possible between the electrodes on the diamond-based detector and the coaxial cables so as not to deform the pulse shape. The pulse width of the output signal can be shortened by using a detector with lower electrical capacitance. The rms noise signal level $\left(\sigma_{n}\right)$ was suppressed to about $0.5 \mathrm{mV}$ for the one-shot measurements.

The linearity of the output signal of the injected beam was also demonstrated as shown in Fig. 5. The number of incident electrons per pulse was estimated using the output charge from the silicon PIN photodiode (Hamamatsu S5377-05). The output charge from the diamond-based detector is proportional to the number of incident electrons per pulse in the range of approximately $10^{3}$ to $10^{7}$ electrons/pulse. The lower detection limit for a single

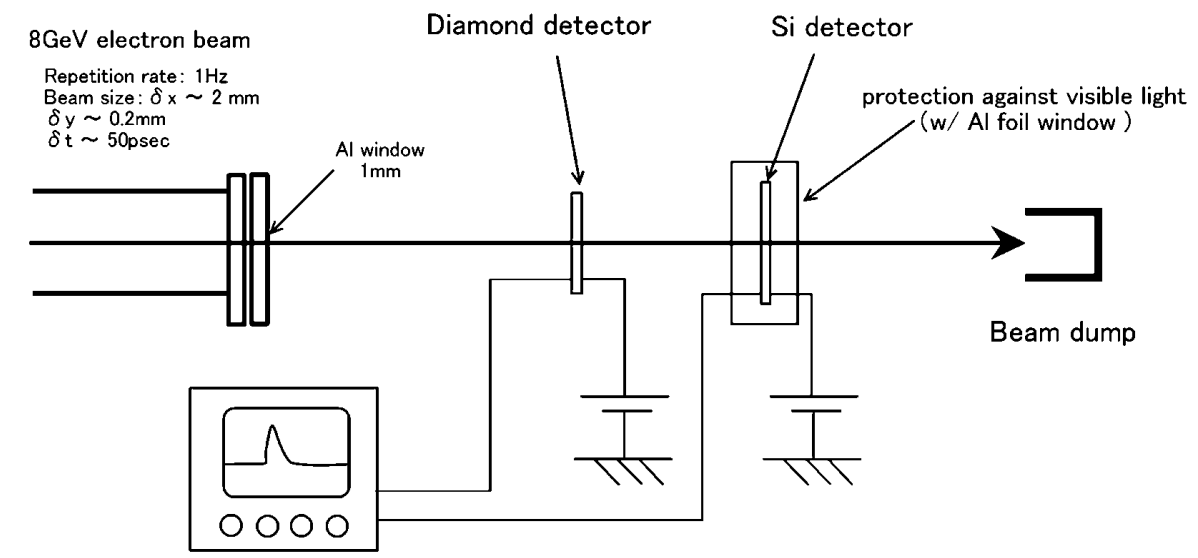

FIG. 3. Setup of the electron beam tests performed in the SPring-8 synchrotron beam dump area. 


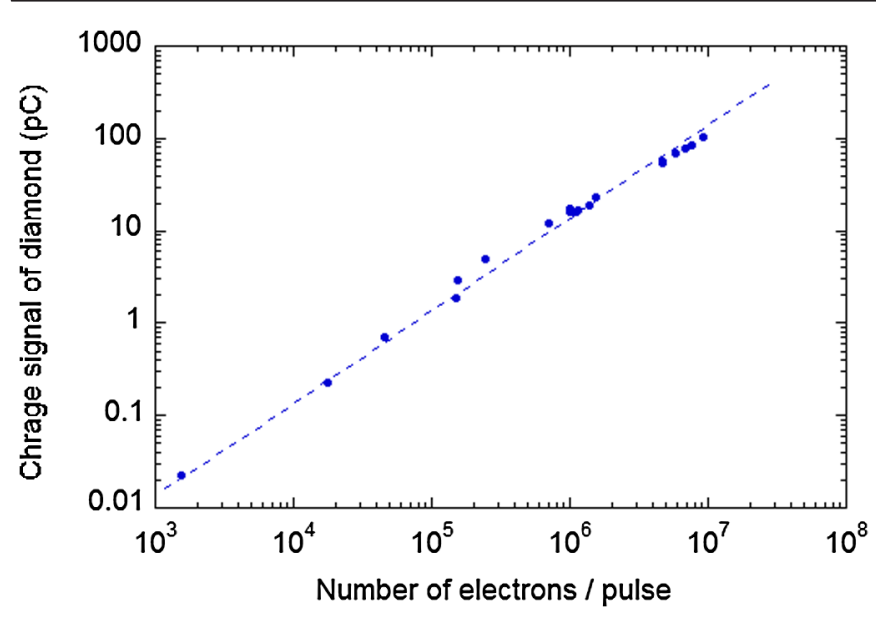

FIG. 5. Linearity of output signal.

shot is about $2 \times 10^{3}$ electrons/pulse, which is equivalent to a charge signal of $25 \mathrm{fC}$ when the bias voltage is $+100 \mathrm{~V}$. Here, we define the pulse height at which the output signal corresponds to $10 \sigma_{n}$ as the lower detection limit.

Assuming the tolerance of demagnetization rate of undulator magnets of $1 \%$ in 10 years, the tolerance of incident electron on the undulator magnets is estimated to be $4 \times 10^{14}$ electrons/10 year, which is based on the experimental results [1]. In the case of the $60 \mathrm{~Hz}$ operation for 24 hours and 365 days, the required lower detection limit corresponds to $2 \times 10^{4}$ electron/pulse. The experimental result satisfies this requirement. Therefore, the interlock for machine protection can be set by the instantaneous dose (shot by shot) as well as the integrated dose.

In the literature the number of electron-hole pairs created by a MIP in diamond is assumed to be 36 per $\mu \mathrm{m}$. In the above case, it is 78 electron-hole pairs per $300 \mu \mathrm{m}$ with the bias voltage of $+100 \mathrm{~V}$, which corresponds to 0.26 electron-hole pairs per $\mu \mathrm{m}$. This result originates the fact that the collection distance in the diamond detector used in this experiment is extremely suppressed. The collection distance can be described as $d=\mu \tau E$, where $d, \mu, \tau$, and $E$ are the collection distance, the mobility, the life time of carriers, and the applied bias voltage, respectively [3]. Increasing the bias voltage, the charge signal of diamond can be enhanced by a factor of several. However, to avoid the surface discharge, and to guarantee steady operation, the electrode bias is set to $+100 \mathrm{~V}$ in our experiments.

\section{DESIGN OF THE BEAM HALO MONITOR}

A beam halo monitor has been prepared to measure the beam halo at the $250 \mathrm{MeV}$ SPring-8 Compact SASE Source (SCSS) test accelerator for SACLA [9]. The diamond-based detectors are fixed on holders as shown in Fig. 6(a). Radio-frequency coaxial cables for ultrahigh vacuum are connected to the electrodes of the detectors using very short wires. Therefore, the pulse width can be shortened, the deformation of pulse shape can be reduced,

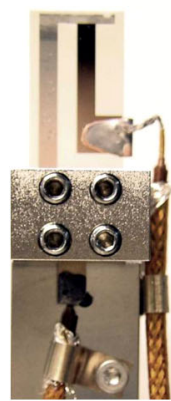

(a)

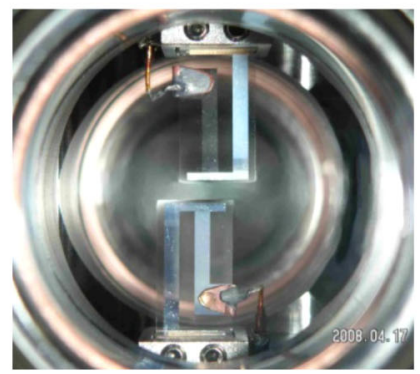

(b)

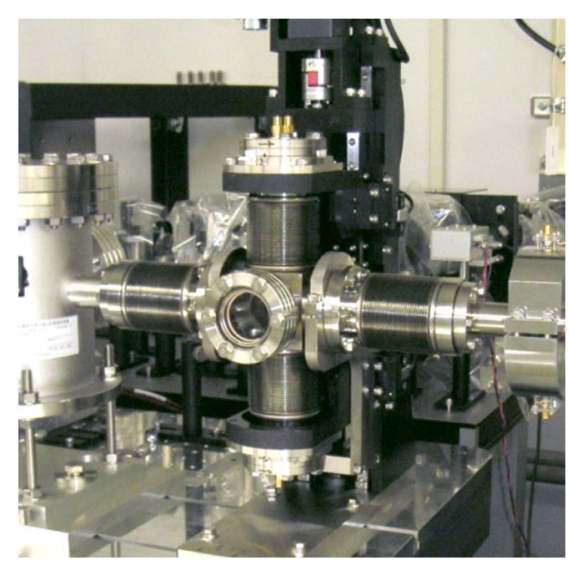

(c)

FIG. 6. Photographs: (a) diamond-based detector on the holder, (b) configuration of the detectors in the vacuum chamber, and (c) beam halo monitor installed at the SCSS test accelerator.

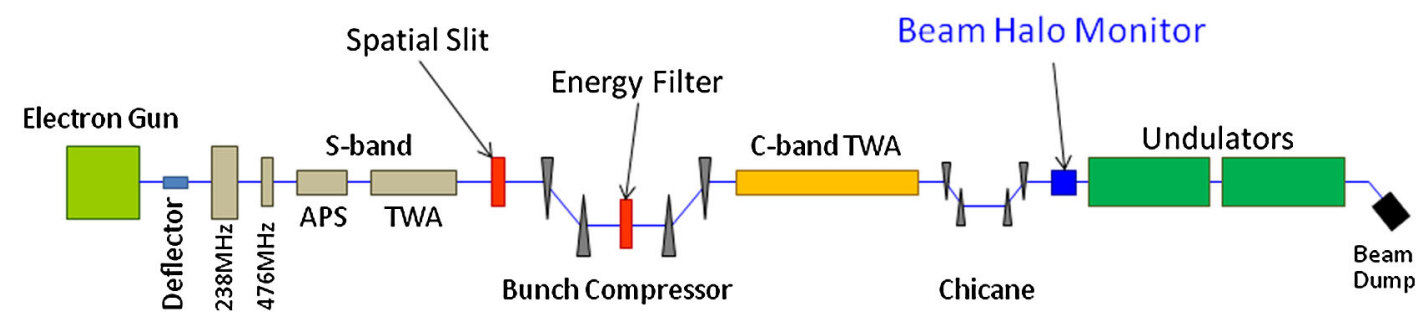

FIG. 7. Layout of SCSS test accelerator. 
and the detection efficiency can be enhanced. Coaxial cables are used for impedance matching. A pair of diamond-based detectors is mounted on the upper and lower sides of the beam center as shown in Fig. 6(b). The gap between the detectors and the center position of the detectors can be changed independently. The core of the electron beam passes between the detectors. The beam halo monitor was installed in front of the undulators at the SCSS test accelerator as shown in Fig. 6(c). Figure 7 shows the layout of the SCSS test accelerator [10]. The spatial slit and energy filter are located after the $50 \mathrm{MeV}$ injector.

\section{FEASIBILITY TESTS}

\section{A. Pulse shape of the beam halo monitor}

Figure 8 shows the pulse shape of the beam halo monitor. The number of incident electrons per pulse was estimated to be about $4 \times 10^{4}$. The active area of the diamond-based detector was irradiated with the core part of the electron beam in this measurement. An output pulse width of $0.4 \mathrm{~ns}$ FWHM was obtained. The pulse width is slightly longer than that measured at the $8 \mathrm{GeV}$ SPring- 8 booster synchrotron, as shown in Fig. 4, because the cable length used in this measurement was $5 \mathrm{~m}$ longer. No significant ringing was observed. Figure 8 also indicates that the effect of the noise source caused by such a thyratron is negligible.

\section{B. Profile measurement of a weak beam}

The charge of a beam was scraped using a spatial slit, and the scanning measurement was performed using both the upper and lower detectors to identify the beam center. Figure 9 shows the obtained profiles of the beam. The profiles measured by the upper and lower detectors are identical. The FWHM of each profile is $1.08 \mathrm{~mm}$, which corresponds to the fact that the height of the active area of the diamond-based detector is $1.0 \mathrm{~mm}$ and the beam diameter is about $0.1 \mathrm{~mm}$. Note that the effective height

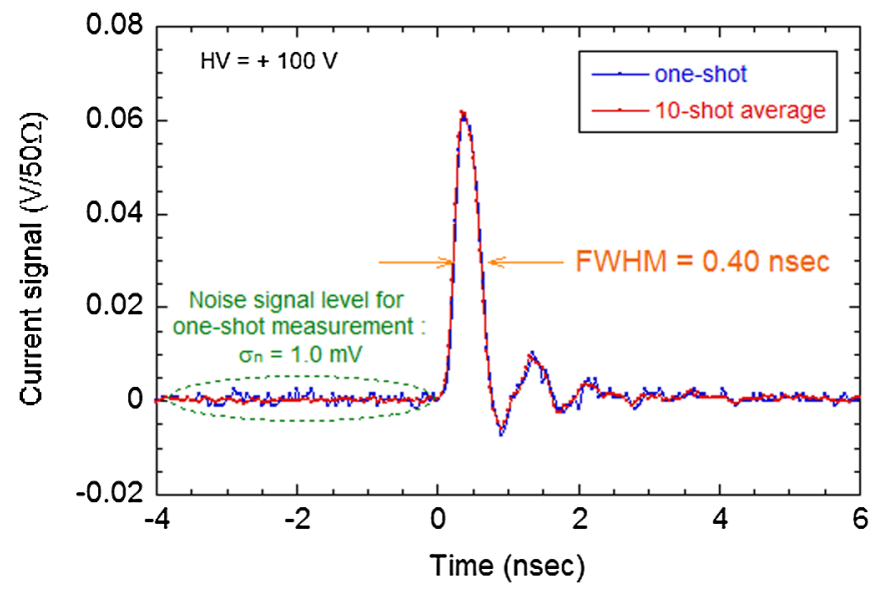

FIG. 8. Pulse shape of the beam halo monitor (blue line: oneshot measurement; red line: 10-shot average).

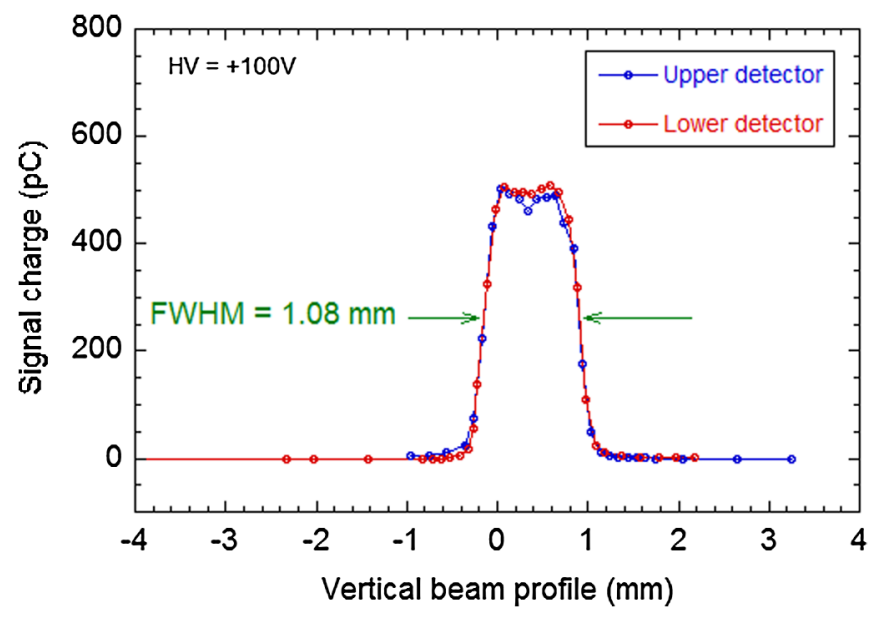

FIG. 9. Profiles of a weak beam (blue line: upper detector; red line: lower detector).

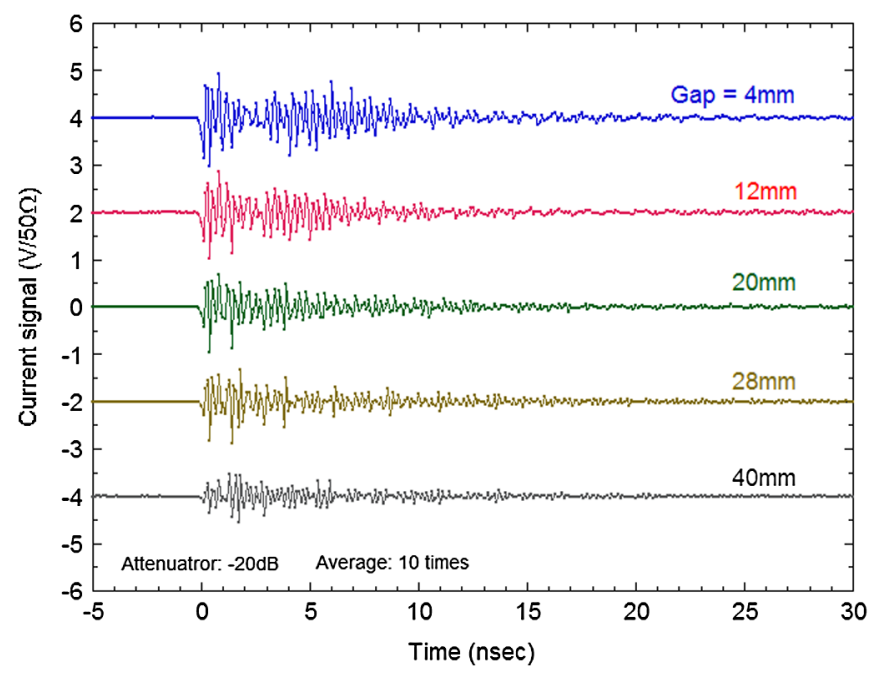

FIG. 10. Waveform caused by induction current. The gaps between the upper and lower detectors are indicated.

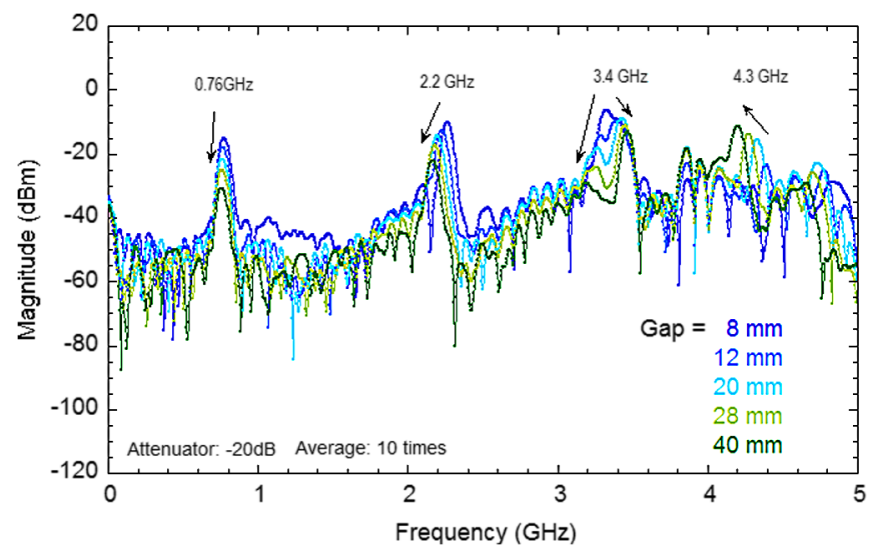

FIG. 11. Spectrum of the signal caused by the induction current. 


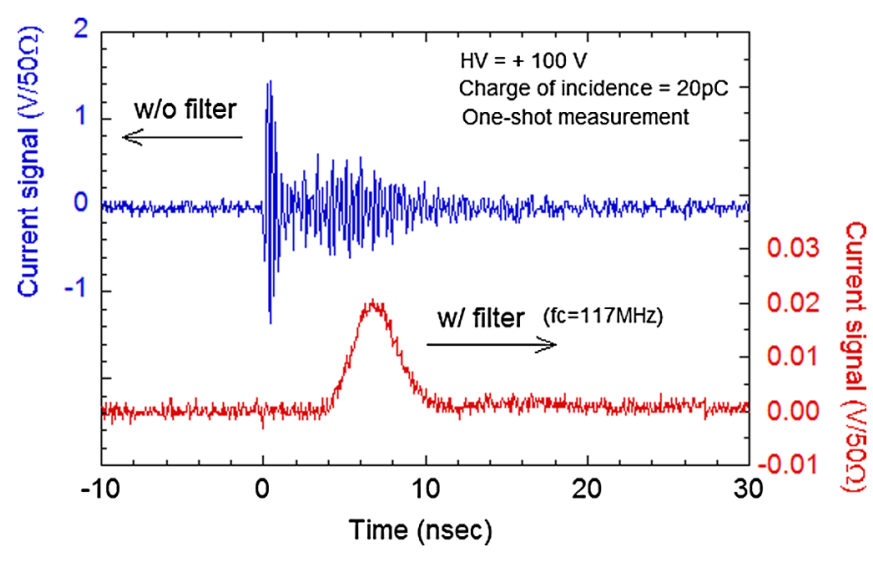

FIG. 12. Effect of induction current before and after suppression. The lower (red) waveform is with LPFs and the upper (blue) waveform is without LPFs.

of the active area is not exactly $1.0 \mathrm{~mm}$, since the electric field may leak at the edge of the electrodes.

\section{Fast-Fourier-transform analysis and filtering}

We evaluated the effect of an induction current $[9,11]$ when an electron beam core with high intensity passes between the detectors. The effect of wakefields was observed with various gaps between the detectors. The typical charge of the beam was $1.2 \times 10^{8}$ electrons/pulse in these measurements.

Waveforms caused by the induction current are shown in Fig. 10. The magnitude of the peaks increases as the gap between the detectors decreases, but the increase is not significant. This indicates that the wakefield spreads in the vacuum chamber. The spectra of the signals in Fig. 10 are shown in Fig. 11. Several peaks can be seen because the vacuum chamber of the monitor has its own resonant frequencies, which change as the actuators of the detectors are trimmed.

We adopted low-pass filters (LPFs) to suppress the effect of the induction current. Figure 12 shows the effect of the induction current before and after its suppression. The lower (red) and upper (blue) waveforms are those with and without LPFs, respectively. The effect of the induction current can be suppressed by using suitable LPFs, and the net signal from electron-hole pairs, which originates from the halo part of the electron beam, can be measured.

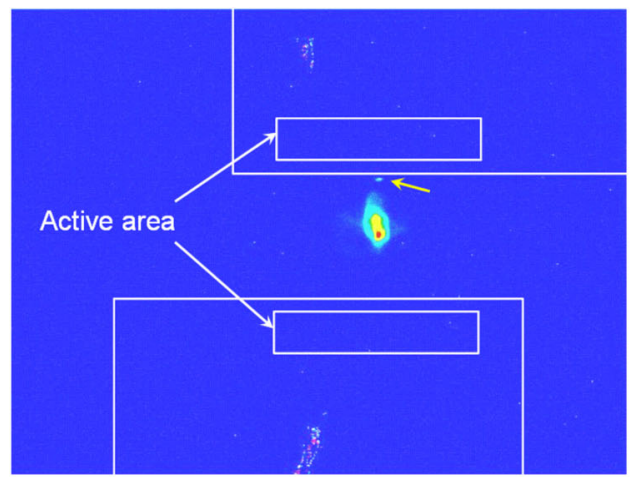

(a) Normal case

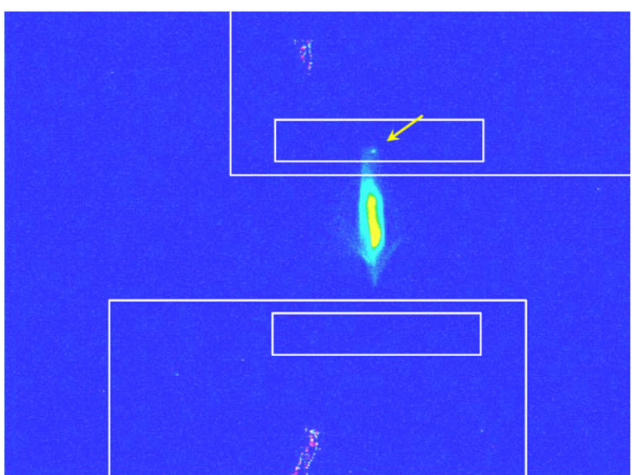

(b) Defocused case

FIG. 13. OTR images. The spots indicated by yellow arrows are due to satellite bunches.

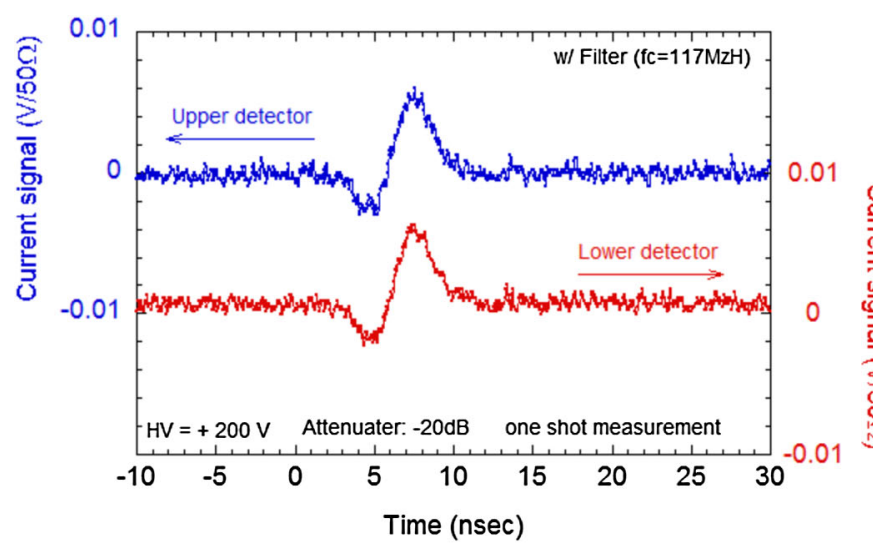

(a) Normal case

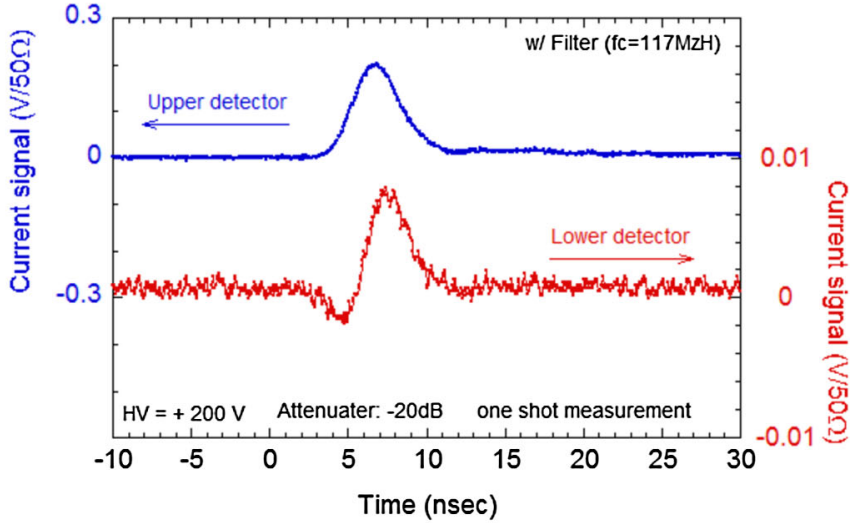

(b) Defocused case

FIG. 14. Signals from the beam halo monitor. 


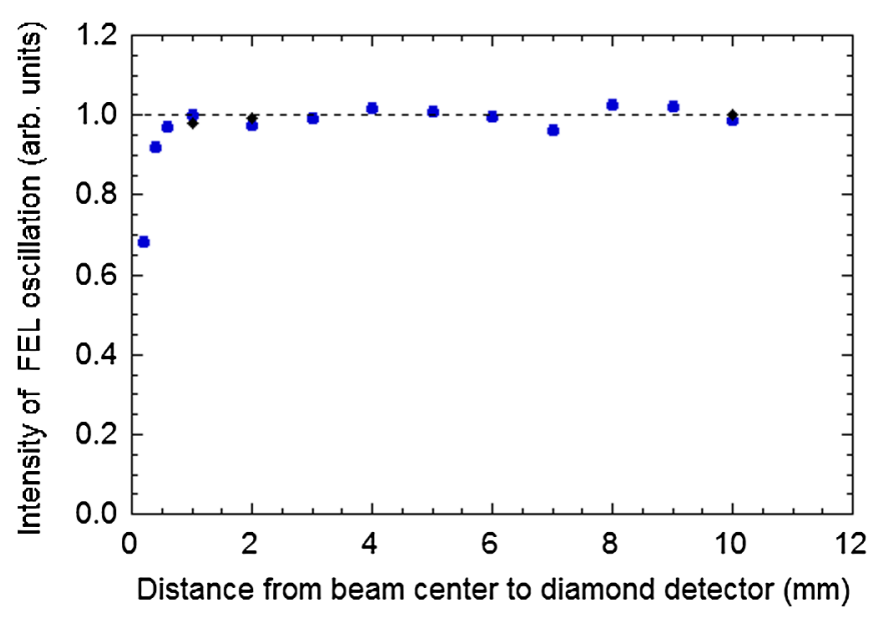

FIG. 15. Effect of distance from beam center to diamond-based detector on FEL oscillation. The intensity of FEL oscillation (vertical axis) was calculated from an output signal of a photodiode.

\section{Observation of a satellite pulse}

The beam was defocused in the vertical direction by adjusting some of the quadrupole magnets, and we attempted to observe the effect of changing the beam size. Figures 13(a) and 13(b) show optical transition radiation (OTR) images of the normal case and the defocused case, respectively. White lines indicate the outlines of the upper/lower detectors, which have a $10 \mathrm{~mm}$ width and an active area of $5 \mathrm{mmH} \times 1 \mathrm{mmV}$. Figures 14(a) and 14(b) show the output signals from the upper and lower detectors in the normal case and defocused case, respectively. For the lower detector (red line), the pulse height was increased by $20 \%$ by defocusing. Even if the size of the beam core is greatly increased, the intensity of the beam halo does not change markedly. However, the pulse height of the upper detector (blue line) increases by a factor of about 40 because a satellite pulse with a relatively high charge may collide with the active area of the upper detector. The pulse height per shot tends to vary by about $50 \%$ when a satellite pulse collides with the active area.

\section{E. Effect of the detectors on FEL oscillation}

We determined the effect on free electron laser (FEL) oscillation when the diamond-based detectors approach the electron beam [9] as shown in Fig. 15. The intensity of FEL oscillation is not affected by the approach of the detectors if the distance from the beam center to each detector is more than $1 \mathrm{~mm}$. There is no interference with user experiments. This result indicates that the beam halo monitor can be used at any time even during user operation at SCSS test accelerator.

\section{OBSERVATION OF THE BEAM HALO}

We performed vertical scanning measurements using the beam halo monitor to evaluate the signal from the

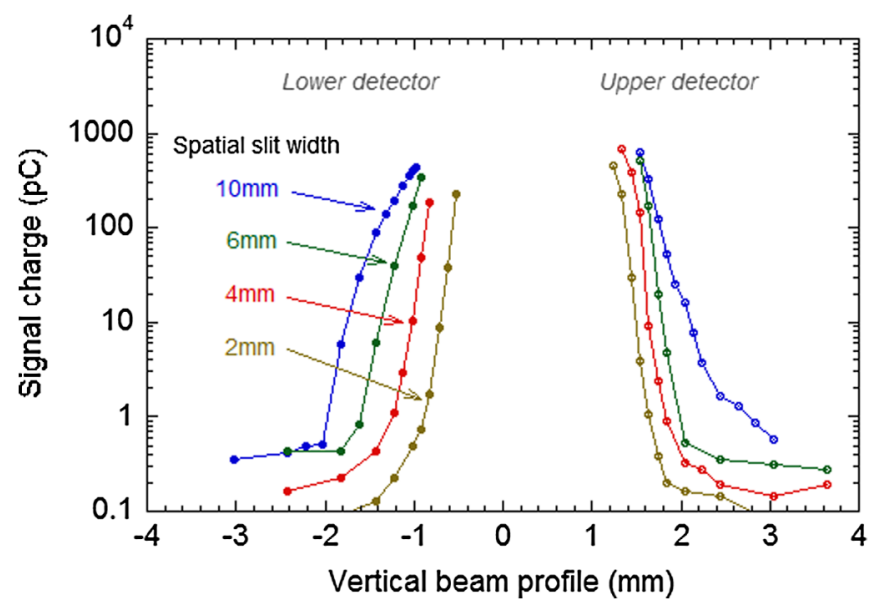

FIG. 16. Scanning measurements for various spatial slit widths. The numbers are the widths of the spatial slit. (The aperture size of the energy filter was fixed to $10 \mathrm{~mm}$.)

bremsstrahlung and secondary electrons produced at the beam ducts and other components. The measurements were carried out with the spatial slit width and energy filter aperture size varied independently.

Figure 16 shows the signal charge as a function of the vertical position of the active area of the diamond-based detector for various spatial slit widths [9]. The width of the spatial slit, which was placed immediately after the end of the injector as shown in Fig. 7, was varied systematically. The signal charge at vertical positions of above $+2 \mathrm{~mm}$ and below $-2 \mathrm{~mm}$ was lower than $1 \mathrm{pC}$ when the slit was fully opened.

Figure 17 shows the signal charge for various aperture sizes of the energy filter. The aperture size of the energy filter, which was placed in the bunch compressor as shown in Fig. 7, was varied systematically. The profiles of the halos did not change, in contrast to the case of changing the width of the spatial slit.

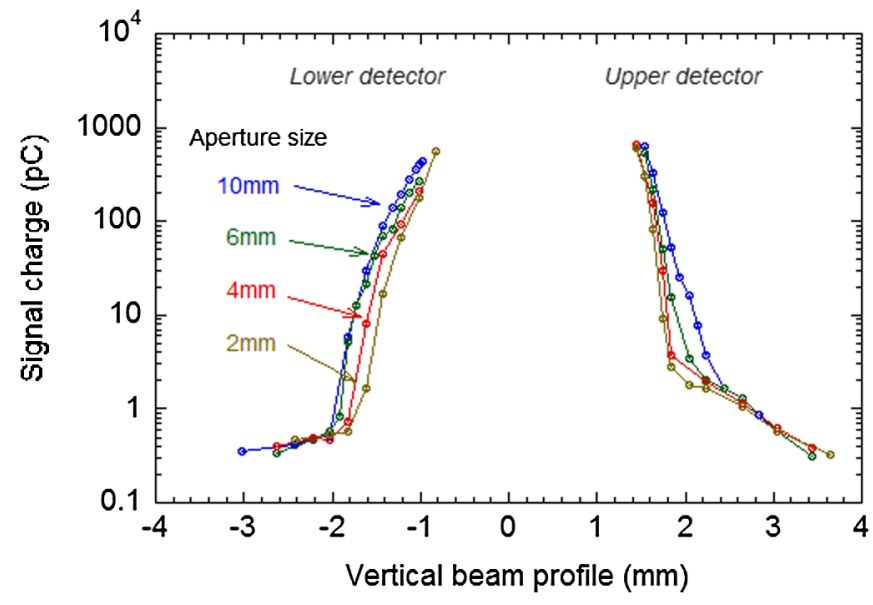

FIG. 17. Scanning measurements with various aperture size of the energy filter. The numbers are the aperture sizes of the energy filter. (The width of the spatial slit was fixed to $10 \mathrm{~mm}$.) 
In both cases discussed above, the profiles of the beam halos are narrow compared with the beam duct of $20 \mathrm{~mm}$ diameter, while the profile due to bremsstrahlung and secondary electrons is expected to be broadened to the diameter of the beam duct. Thus, we conclude that the signal from the bremsstrahlung and secondary electrons is negligibly small in the SCSS test accelerator.

\section{OPERATIONAL EXPERIENCE}

\section{A. Instrumentation}

We prepared a preamplifier for the beam halo monitor and used a synchronous data acquisition system to confirm the medium/long-term stability of monitoring as shown in Fig. 18. The preamplifier was originally developed for the current transformer in the SCSS test accelerator to measure the beam current accurately [12]. It suppresses the noise with a frequency of a few $\mathrm{MHz}$ emitted from the thyratron of the klystron modulator and the ringing noise caused by the wakefield of the electron beam. The time constant of the amplifier was adjusted to about $40 \mathrm{~ns}$ for this purpose. The input terminals were modified to match the single-ended output of the beam halo monitor. An

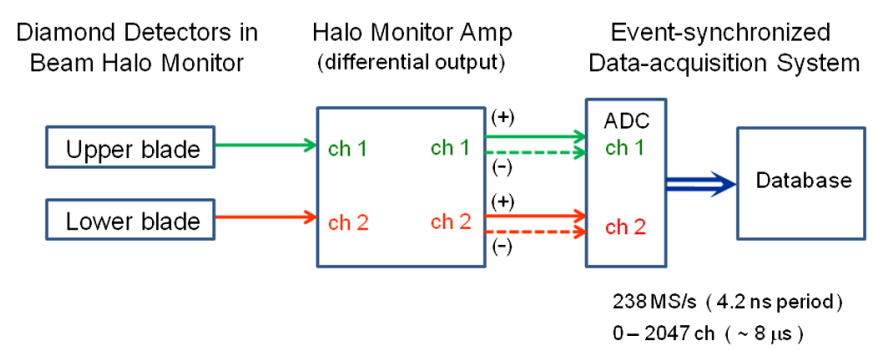

FIG. 18. Schematic diagram of the beam halo monitoring system.

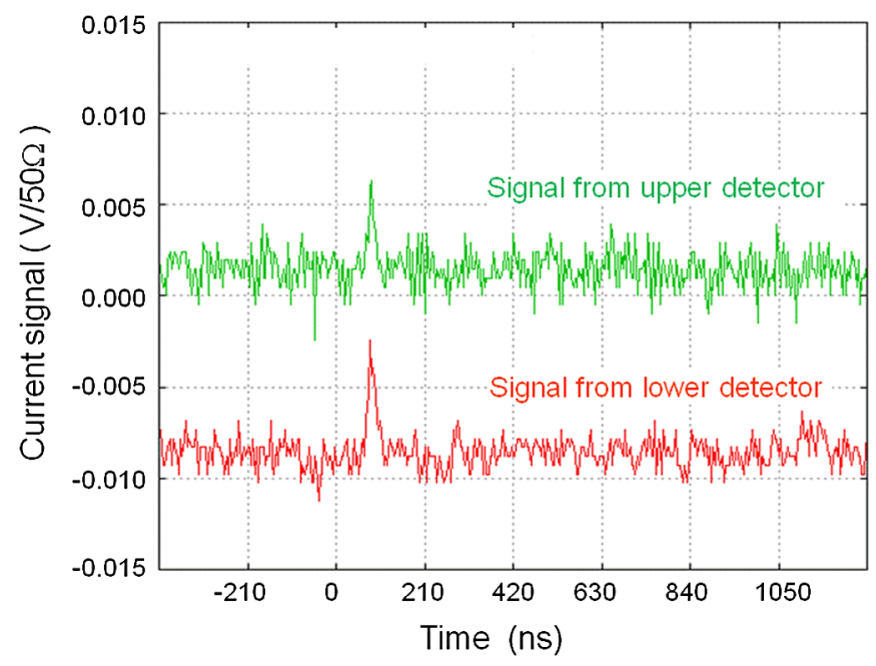

FIG. 19. Typical waveform stored in database. The upper (green) waveform is from the upper detector and the lower (red) waveform is from the lower detector. event-synchronized data acquisition system was installed in the control system of the SCSS test accelerator [13]. This system can record a set of data from rf signals and beam monitor signals that are synchronized with the same electron beam shots. In the case of the beam halo monitor, waveform data at a rate of $238 \mathrm{MS} / \mathrm{sec}$ and integral value (charge) data for all shots are continuously stored in a database. Figure 19 shows a typical waveform stored in the database during the user operation [14]. The gap between the detectors is $5 \mathrm{~mm}$.

\section{B. Stability tests}

Figure 20 shows the result of a half-day measurement during the period of machine study. The charge signal from

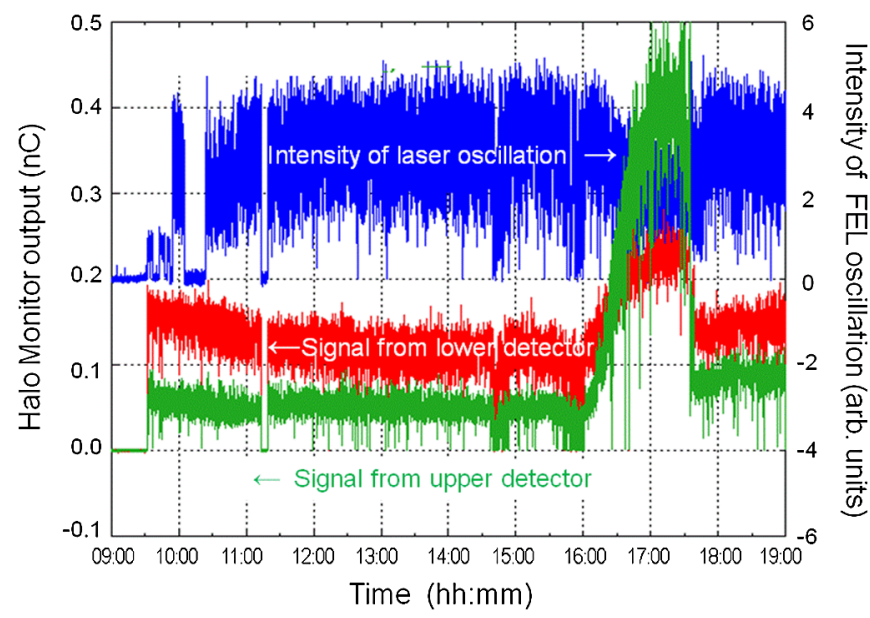

FIG. 20. Result of half-day measurement during the machine study period. The left axis shows the charge signals from the upper (green) and lower (red) detectors of the beam halo monitor, and the right axis shows the intensity of the FEL oscillation (blue). The gap between the detectors is $4 \mathrm{~mm}$.

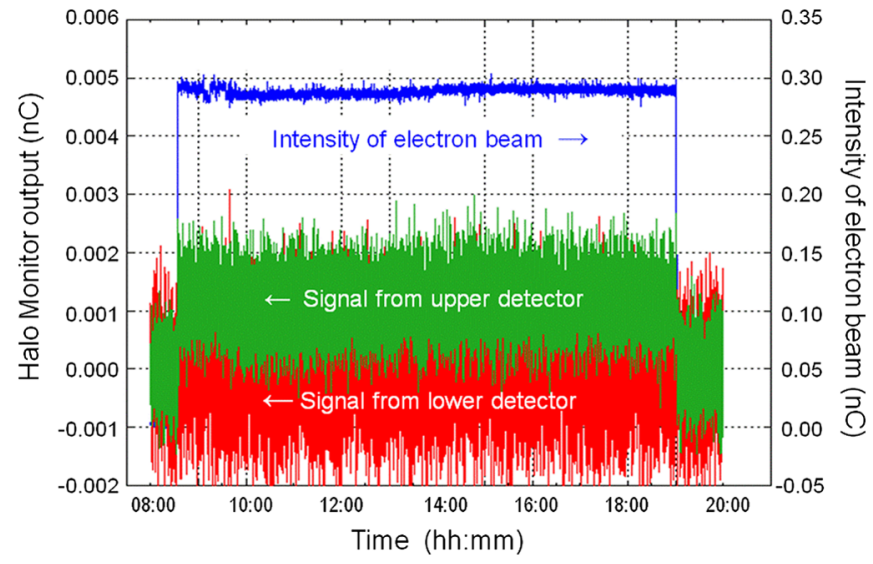

FIG. 21. Result of stability test. The left axis shows the integral output signal of the beam halo monitor (upper: green; lower: red), and the right axis shows the intensity of the electron beam (blue). The gap between the detectors is $4 \mathrm{~mm}$. 
each diamond-based detector was calculated by integration of the pulse shape. During the machine study, machine conditions were continuously changing. At the beginning of the machine study, the intensity of the FEL oscillation was optimized, and the charge signal of the beam halo monitor tended to decrease. In the period 16:00-17:30, the phase of the rf cavity was trimmed and the integral output signal was increased.

Figure 21 shows the result of a stability test during the user operation. The machine operation usually starts at $8: 30$, and is offered to users soon after a quick beam tuning. The machine operation stops at 19:00. The output signal of the beam halo monitor was very stable for about 10 hours. These observation results indicate that the beam halo monitor system operates effectively.

In order to use the beam halo monitor during the user operation for years, the detectors must have radiation hardness. Radiation damage was not significant after the halo monitor was used at the SCSS test accelerator for one year or more. The extremely high radiation hardness of the diamond detector has already been demonstrated in other facilities $[15,16]$. Our next issue is to evaluate the radiation damage quantitatively.

\section{CONCLUSION}

We have developed an electron beam halo monitor using diamond-based detectors to prevent the demagnetization of the ID permanent magnet by electron beam irradiation. A feasibility test was performed at the beam dump of the $8 \mathrm{GeV}$ SPring- 8 booster synchrotron and the $250 \mathrm{MeV}$ SCSS test accelerator for SACLA. We demonstrated that the output charge of the diamond-based detector is proportional to the number of incident electrons per pulse in the range of $2 \times 10^{3}$ to $10^{7}$ electrons/pulse. The effect of environmental noise in the klystron gallery is suppressed so that it is not observed. The effect of the induction current can be controlled by using low-pass filters. No effect of the secondary electrons or the radiation was observed in a test performed at the SCSS test accelerator. There was no effect on free electron laser oscillation even when the diamond-based detectors were placed at a distance of a few $\mathrm{mm}$ from the beam axis. We prepared a preamplifier for the beam halo monitor and used a synchronous data acquisition system to confirm the medium/long-term stability of monitoring. During the user operation, the output of the beam halo monitor is very stable. All these results suggest that the electron beam halo monitor is feasible for use as an interlock sensor to protect the undulator permanent magnets used in SACLA from radiation damage.

\section{ACKNOWLEDGMENTS}

We would like to thank the SCSS Test Accelerator Operation Group for their comments on beam tests, and S. Takahashi and A. Watanabe of JASRI/SPring-8 for their support in the preparation of the hardware. The beam tests using the diamond-based detector carried out at the beam dump of the SPring-8 booster synchrotron were supported by K. Fukami, T. Aoki, and S. Suzuki of JASRI/SPring-8. This work was developed by the Safety Design Group of XFEL/SPring-8, and was partly supported by Japan Society for the Promotion of Science, Grant-in-Aid for Scientific Research (c) 21604017.

[1] T. Bizen et al., Nucl. Instrum. Methods Phys. Res., Sect. A 574, 401 (2007).

[2] R. J. Tapper, Rep. Prog. Phys. 63, 1273 (2000).

[3] L. S. Pan et al., J. Appl. Phys. 74, 1086 (1993).

[4] L. Fernandez-Hernando et al., Nucl. Instrum. Methods Phys. Res., Sect. A 552, 183 (2005).

[5] E. Griesmayer et al., in Proceedings of the 2010 Beam Instrumentation Workshop (Jefferson Lab, Santa Fe, 2010), MOCNB02.

[6] H. Aoyagi et al., AIP Conf. Proc. 705, 933 (2004).

[7] H. Aoyagi et al., in Proceedings of the 22nd Workshop on Radiation Detectors and Their Uses, KEK Proc. No. 200814 (KEK, Tsukuba, Japan, 2008), p. 108.

[8] H. Aoyagi et al., in Proceedings of the 11th European Particle Accelerator Conference, Genoa, 2008 (EPS-AG, Genoa, Italy, 2008), THPC146.

[9] H. Aoyagi et al., in Proceedings of the 9th European Workshop on Beam Diagnostics and Instrumentation for Particle Accelerators (Paul Scherrer Institut, Basel, Switzerland, 2009), TUPB24.

[10] H. Tanaka et al., in Proceedings of the 11th European Particle Accelerator Conference, Genoa, 2008 (Ref. [8]), WEOAM01.

[11] H. Aoyagi et al., in Proceedings of the 24th Workshop on Radiation Detectors and Their Uses, KEK Proc. No. 201010 (KEK, Tsukuba, Japan, 2010), p. 73.

[12] A. Higashiya, H. Maesaka, and Y. Otake, in Proceedings of the 4th Annual Meeting of Particle Accelerator Society of Japan and the 32nd Linear Accelerator Meeting in Japan (PASJ, Wako, Japan, 2007), WP45, p. 339.

[13] M. Yamaga et al., in Proceedings of the 12th International Conference on Accelerator and Large Experimental Physics Control Systems (SPring-8, Kobe, Japan 2009), TUB003.

[14] H. Aoyagi et al., in Proceedings of the IPAC'10 Conference, Kyoto, Japan (ICR, Kyoto, 2010), WEPEB068.

[15] C. Bauer et al., Nucl. Instrum. Methods Phys. Res., Sect. A 367, 207 (1995).

[16] T. Behnke et al., Nucl. Instrum. Methods Phys. Res., Sect. A 489, 230 (2002). 